\title{
NUEVAS VIÑAS EN CHILE CENTRAL: 1850-1900 ${ }^{1}$
}

\author{
FÉLIX BRIONES QUIROZ ${ }^{2}$
}

\section{RESUMEN}

A partir de mediados del siglo XIX se experimenta en Chile el surgimiento de una nueva actividad económica en la región central del país, la llamada "viña francesa". La mayor concentración de este rubro estuvo en los alrededores de Santiago, específicamente en el llamado Llano del Maipo. Hacia el sur la existencia de los nuevos viñedos fue de menor envergadura, aunque no menos importante, pues existían las condiciones necesarias para su desarrollo. Las nuevas viñas se basaron en la vitivinicultura francesa, porque principalmente desde Francia se importaron las plantas, las formas de cultivos, la tecnología e incluso, los propietarios de estos viñedos contrataron técnicos franceses para dirigir las labores que requería esta nueva actividad. Al mismo tiempo, construyeron los edificios e instalaciones, entre los que se destacaban la casa habitación con su parque y las bodegas subterráneas en donde se guardaban los vinos que más tarde serían puestos en el mercado nacional e internacional. Por su parte la preocupación del Estado se manifestó a través de disposiciones legales tendientes a proteger las viñas chilenas de las enfermedades, por la contratación de profesores especialistas franceses que se desempeñaron en el Instituto Agrícola y las diversas escuelas agrícolas del país. También, por medio de la Quinta Normal de Agricultura, las diversas comisiones de estudio realizadas tanto en Chile como en el extranjero. Fue a partir de la década de 1880, y tras la incorporación de las provincias mineras del norte, Tarapacá y Antofagasta y, en el sur, de la Araucanía, que se dinamizó aún más la producción de vino y su comercialización, en el mercado interno. Es más, se constituyó en la salvación de los viñateros nacionales, pues pasó a ser más importante que el mercado internacional, en el que la demanda del producto chileno era limitada.

Palabras claves: Vitivinicultura, viñas nuevas, viñas francesas.

\section{ABSTRACT}

Beginning in the mid 19th century, a new economic activity emerged in Central Chile: the French vineyard. The largest concentration of this activity was in the agricultural areas outside of Santiago, specifically in the Llano del Maipo (Maipo Valley). Towards the south, the new vineyards were smaller but no less important because the conditions necessary for their development existed. The new vineyards were based in French vitiviniculture because the plants, cultivation techniques, and technology were principally imported from France. Indeed, even French technicians were hired to direct the labors of this new activity. At the same time, they built buildings and installation including the principal house with its park and subterranean cellars for the wine to be sold in the international and national market. State involvement was present in the legal dispositions to protect Chilean wines form diseases, the hiring of French specialists for the Agricultural Institute and other agricultural schools in Chile. Additionally, through the Quinta Normal de Agricultura, diverse study commissions were held both in and outside Chile. Beginning in the $1880 \mathrm{~s}$, and after the incorporation of the northern mining provinces, Tarapacá and Antofagasta, and the Araucanía in the south, wine production and its commercialization in the Chilean market became more dynamic. Indeed, it was the salvation of national winemakers, becoming more important than the international market where there was limited demand for Chilean wine.

Keywords: Vitiviniculture, new vineyards, French vineyards.

\section{INTRODUCCION}

La expresión "nuevas viñas en Chile central" significa el surgimiento de una nueva actividad económica en el campo chileno, teniendo como modelo a la vitivinicultura francesa. La localización geográfica de las viñas francesas comprendía desde el río Aconcagua por el norte hasta el Maule por el sur, concentrándose en el Llano del Maipo.

\footnotetext{
Ponencia presentada en las XI Jornadas de Historia Regional de Chile organizadas por el Departamento de Ciencias Históricas y Sociales de la Facultad de Humanidades y Arte de la Universidad de Concepción, entre los días 18 y 21 de octubre de 2004.

2 Doctor en Historia por la Universidad de Sao Paulo, Brasil. Profesor de la Escuela de Historia y Geografia, Departamento de Ciencias Sociales, Facultad de Educación y Humanidades, Universidad del Bío-Bío, Chillán (Chile).
} 
La creación de viñas con vid francesa en Chile permitió a los viticultores cambiar sus métodos de cultivo y de fabricación de vino, adoptando los que se practicaban en las regiones de donde procedían las nuevas plantas. El resultado de este esfuerzo fue un gran número de viñas francesas y la producción de vinos sanos y de excelente calidad, que podían competir con los productos análogos del viejo mundo ${ }^{3}$.

Las nuevas variedades se fueron multiplicando para satisfacer las necesidades del país. Esta situación fue percibida por el director de la Quinta Normal de Agricultura, Luis Sada di Carlo, que comenzó a cultivar variedades de vid con el propósito de multiplicarlas y abastecer a quienes las necesitaban. También, propietarios de chacras y haciendas solicitaron plantas de vid de las distintas variedades y contrataron personas especializadas desde Francia, que por su práctica y experiencia contribuyeron a hacer de esta industria un ramo nuevo y de gran interés ${ }^{4}$.

En Chile existían viñedos dirigidos por personas idóneas, que perfeccionaron el cultivo de la vid en las provincias centrales. De tal forma, que la industria vitivinícola cobró un vigoroso impulso gracias a las nuevas viñas y al trabajo realizado por los franceses encargados de dirigir las labores que ellas requerían ${ }^{5}$.

El presente artículo es una visión general del surgimiento de una nueva actividad económica en la región central de Chile: se trata de la nueva vitivinicultura chilena a partir de mediados del siglo XIX. En especial, se destacará la influencia francesa, la creación y existencia de nuevas viñas, propietarios y extensión de los viñedos.

\section{CHILE: LA FRANCIA DE AMERICA DEL SUR}

A partir de mediados de la década de 1840 viñateros procedentes de la región de Charentes, en Francia, introdujeron las cepas más finas ${ }^{6}$. La primera viña francesa fue plantada en Chile por M. Nourrichel en 1845. Esta viña, llamada La Luisa, formaba parte de la chacra Vigouroux, que más tarde fue incorporada a la Quinta Normal de Agricultura?.

Fue a mediados del siglo XIX que la vitivinicultura chilena entró en un período evolutivo debido a la introducción de sarmientos franceses. Se dice que uno de los primeros en traer plantas desde Francia fue M. Bertrand, viticultor contratado por Silvestre Ochagavía ${ }^{8}$. También se señala que José Tomás Urmeneta trajo las primeras plantas de vid francesa, de tal forma, que la viña "Urmeneta" sería la pionera en la elaboración de vino tipo francés en Chile?.

A mediados de la década de 1870 se manifestaba que "en los últimos diez o quince años, la viticultura se ha transformado y propagado con mucha rapidez"10. Esta situación se debió a que unos cuantos hacendados progresistas comenzaron a mejorar el cultivo de la vid y el defectuoso sistema de vinificación ${ }^{11}$.

Las viñas de Chile central, en especial las de vid francesa, fueron la culminación de un proceso iniciado antes de la década de 1850 y que a partir de la década de 1870 cobró un vigoroso impulso debido a la contratación de un especialista francés, René F. Le Feuvre, para hacerse cargo de la dirección del Instituto Agrícola, dependiente de la Quinta Normal de Agricultura" ${ }^{12}$ Su objetivo era hacer de Chile "la Francia de América del Sur", desde el punto de vista de la vitivinicultura ${ }^{13}$. De tal forma, que "la generalización de las cepas francesas en Chile se debió en gran parte a M. Le Feuvre, director de la Quinta Normal de Agricultura. Es él quien dando a conocer la planta, desde hace 17 años, es él quien enseñando su cultivo, es él, en fin, quien con una activa propaganda, en la cátedra y fuera de ella, ha impuesto la innovación a los agricultores"14.

3 Séve, Eduardo.1876. "La patria chilena". Boletín de la Sociedad Nacional de Agricultura. Santiago: No 22,5 de septiembre. pp. 545-546.

4 Gay, Claudio. 1865. Historia fisica y politica de Chile. Imprenta de E. Thunot y Cía., París . Tomo II. p. 175.

Ibid., pp. 200-204.

Blancpain, Jean Pierre. 1987. Francia y los franceses en Chile (1700-1980). Editorial Universitaria, Santiago. p. 212.

7. Vega E., M. 1904. Album de la Colonie Francaise au Chile. Imprimerie et Lithographie Franco-Chilienne, Santiago. p. 205.

8 Encina, Francisco A. Historia de Chile (1940 - 1952). Editorial Ercilla, Santiago, 1984. Tomo XXVI. p. 130; León, Víctor E. 1947. Uvas y vinos de Chile. PLATT, Establecimientos Gráficos S.A., Buenos Aires. p. 55.

- Valle, Carmen. 1954. Don Maximiano. Editorial Alonso de Ovalle, Santiago. p. 164.

"Séve, Eduardo 1876. "La patria chilena", Boletín de la Sociedad Nacional de Agricultura. op. cit. p. 545.

"Menadier, Julio. 1877. "Sociedad Nacional de Vinicultores". Boletín de la Sociedad Nacional de Agricultura. Santiago: No 14,5 de marzo. p. 257.

12 Memoria del Ministerio de Relaciones Exteriores y de Colonización presentada al Congreso Nacional de 1874 . p. 531 ; Datos biográficos de los ex directores del Instituto Agrícola de Chile, señores René F. Le Feuvre y Julio Besnard. Boletín de la Sociedad Agricola del Sur. Concepción: No 23, 8 de diciembre de 1904. pp. 688-689; Rojas L., Manuel. 1891. Tratado de viticultura y vinificación. Imprenta de La Libertad, Talca. p. 8.

${ }^{13}$ Le Feuvre, René F. 1876. "Empalizamiento de las viñas". In: Primer congreso libre de agricultores de la República de Chile en 1875 . Imprenta de la República de Jacinto Núñez, Santiago, septiembre. p. 273; Idem., "Informe sobre el estado de las viñas en San Felipe, Los Andes, Mendoza, San Juan y Buenos Aires". Boletín de la Sociedad Nacional de Agricultura. Santiago: N 16, 5 de junio de 1884 . p. $346 ;$ Durán C., Horacio. 1894. "La viña del pais alambrada". Boletín de la Sociedad Nacional de Agricultura. Santiago: N ${ }^{\circ} 11,20$ de junio. p. 439.

${ }^{14}$ Rojas L., Manuel. Tratado de viticultura y vinificación. op cit., p. 8. 
Este proceso continuó en los años posteriores y las nuevas viñas se propagaron rápidamente por el centro del país. Así, en los inicios de la década de 1890 el cultivo de la vid había experimentado un gran desarrollo, las antiguas viñas fueron reemplazadas por las de parras francesas, obteniéndose un producto de mejor calidad. Anualmente, se plantaron cientos y miles de hectáreas a la francesa y fue perdiendo importancia el antiguo sistema, experimentándose una revolución en el campo chileno, pues las viñas francesas se plantaban por todas partes y la antigua plantación se fue extinguiendo poco a poco ${ }^{15}$.

Las viñas francesas constituyeron una nueva actividad en el campo chileno. Para su formación se requería de nuevos conocimientos e incorporación de tecnología con el fin de obtener un producto de calidad capaz de competir tanto en el mercado nacional como internacional con los elaborados por otros países y regiones vinícolas del mundo, tales como España, Portugal, Italia, Alemania, California y Francia ${ }^{16}$.

Los propietarios de viñas francesas adquirieron, especialmente en Francia, maquinaria, herramientas y utensilios que se necesitaban para iniciar la nueva actividad económica e incluso trajeron los encargados de dirigir las diversas labores que requerían. Así, se fueron originando las nuevas viñas especializadas y prácticamente hicieron desaparecer las que existían desde los tiempos de la Colonia, en especial, en los alrededores de la ciudad de Santiago ${ }^{17}$. Sin embargo, todavía a fines del siglo XIX quedaban en esta zona algunas hectáreas de la llamada viña país. Por ejemplo, en el viñedo de Macul, en 1894 había tres hectáreas ${ }^{18}$; en 1897, José Palma Guzmán poseía 7 has., Francisco Undurraga, 10 has, E. Cotidio Fernández, 12,50 has., Enrique Morandé, 21,87 has. ${ }^{19}$.

La incorporación de nuevos métodos de cultivo y de tecnología se aprecia, por ejemplo, en Macul, donde existían variedades de vid francesa tales como cabernet que constituían el $60 \%$ y otras como pinot, verdot, cot-rouge, merlot que conformaban el $40 \%$ restante. Estaban plantadas en hileras y los postes eran de fierro. Para el riego se aprovechaba el agua del canal las Perdices, ramal del canal del Maipo, y se realizaba cada quince días. Toda la viña estaba cerrada por sólidas y altas murallas de adobe. Los cuarteles en donde se plantaba la vid eran de $40 \times 40$ metros y de $60 \times 60$. Estaban separados por caminos que se cortaban en ángulo recto, de seis a siete metros de ancho provistos de una red de ferrocarril portátil para los variados servicios. En especial, se utilizaba para transportar la uva durante la vendimia hasta la bodega, donde estaban las cubas, la prensa y la máquina vendimiadora ${ }^{20}$.

Las bodegas medían 200 metros de largo por 10 de ancho y eran de dos pisos. El inferior o subterráneo se componía de un amplio departamento, y otro pequeño para los vinos añejos encajonados, constituidos por gruesos muros de cal y ladrillo y una bóveda del mismo material. El piso superior o a nivel del suelo constaba de un vestíbulo al centro y dos extensas bodegas a los lados de cal y ladrillo cubierto con una techumbre de zinc. Las cubas eran de roble americano de 300 arrobas de capacidad. La vasija de guarda era de la misma madera de $6,10,20$ y 30 arrobas, siendo esta última la más común. Anualmente, la producción era de 400 arrobas de chacolí, 6.000 de vino tinto, 600 de vino blanco y una cantidad no especificada de aguardiente ${ }^{21}$.

En algunas haciendas y viñedos existían talleres de tonelerías para fabricar sus envases, como en Viluco, San Nicolás y $\mathrm{Macul}^{22}$. Pero, al mismo tiempo, encontramos, en las diversas ciudades del país, talleres de tonelería que abastecían a los propietarios de viñas ${ }^{23}$.

is Ibid., p. 7.

${ }^{16}$ Estadística Comercial de la República de Chile, 1844-1900.

Memoria del Ministerio de Industria y Obras Públicas presentada al Congreso Nacional en 1897. Op cit., pp. $155-163$.

${ }^{18}$ Durán C., Horacio. 1894. "El viñedo de Macul". Boletín de la Sociedad Nacional de Agricultura. Santiago: No 18,20 de septiembre. p. 659.

${ }^{19}$ Memoria del Ministerio de Industria y Obras Públicas presentada al Congreso Nacional en 1897. Op cit., p. 158. De acuerdo a esta fuente, en los departamentos de Santiago, Victoria, Melipilla y Maipo había 90,31 hectáreas de viña país.

${ }^{20}$ Durán C., Horacio 1894. "El viñedo de Macul". Boletín de la Sociedad Nacional de Agricultura. Op cit. pp. 659-660. Para otras viñas ver: Pescheux, Marin. 1870. "Exposición sobre el cultivo de las viñas de la hacienda de Limache y sus productos". Boletín de la Sociedad Nacional de Agricultura. Santiago: No 9, 20 de febrero. pp. 171-172; Durán, Horacio. 1891. "El viñedo de Pachacama". Boletín de la Sociedad Nacional de Agricultura. Santiago: $N^{\circ} 23,5$ de diciembre. pp. 735-743.

${ }^{21}$ Durán C., Horacio 1894. "El viñedo de Macul". Boletín de la Sociedad Nacional de Agricultura. Op. cit., pp. 661-662. Para ver descripciones de bodegas: Rojas L., Manuel. "Viticultura y vinificación". Op. cit., pp. 774-805; Idem. "Tratado de viticultura y vinificación". Op. cit., pp. $41-42$ y 452-458; "La bodega de Macul”. Boletín de la Estación Enológica de Chile. Santiago: $\mathrm{N}^{\circ}$ 3, octubre de $1909 . \mathrm{pp} .34-44$.

${ }^{22}$ Menadier, Julio. 1872. "La hacienda de Viluco". Boletin de la Sociedad Nacional de Agricultura. Santiago: No 10, 5 de marzo. p. 209; Tornero, Recaredo S. 1872. Chile Ilustrado. Guia descriptiva del territorio de Chile, de las capitales de provincia y de los puertos principales. Librería y Agencias del Mercurio. p. 434; Salazar, Gabriel. 1989 Labradores, peones y proletarios. LOM Ediciones, Santiago, 2000. p. 164.

${ }^{23}$ Estadística industrial. 1884 "Nóminas de las industrias del país que sirven de base para la estadística industrial". Boletin de la Sociedad de Fomento Fabril. Vol. I, 25 de febrero. pp. 54-60; 10 de marzo de 1884, pp. 68-73; 14 de abril de 1884 , pp. 108-109; 10 de mayo de 1884 , p. 148. 
Junto con la plantación de las nuevas viñas se fueron construyendo las casas, parques y bodegas, siguiendo el modelo de los viñedos franceses, contratando para ello a "diseñadores franceses"24.

El abastecimiento de maquinaria y utensilios importados para las nuevas viñas podemos apreciarlo a través de los datos proporcionados tanto por los registros de importación como por las casas comerciales que ofrecían a los interesados la variedad que necesitaban para desarrollar sus actividades y trabajos en los viñedos. Los viñateros chilenos importaron duelas y vasijas de roble, corcho para tapar botellas, damajuanas y botellas vacías, lacre para sellar los envases. Además, máquinas para vendimiar, destilar, tapar botellas, embotellar el vino ${ }^{25}$. E incluso, las fundiciones chilenas ofrecían todo tipo de maquinaria y herramientas que se requerían para la nueva vitivinicultura ${ }^{26}$.

Contribuyó al desarrollo de esta nueva actividad la preocupación del Gobierno a través de disposiciones legales, como la de 1874 que prohibió la internación de planta de vid francesa y, tres años más tarde, en 1877 decretó la prohibición de importar al país vid extranjera. Estas medidas fueron las herramientas legales de que dispuso el Gobierno y las autoridades aduaneras para impedir que entrara al territorio nacional una de las plagas más devastadoras de las viñas, se trata del insecto Philloxera vastatrix ${ }^{27}$. De tal forma que la mayoría de las viñas francesas en Chile se formaron con plantas de vid internadas al país desde Francia y, a través del comercio interno de plantas, se propagaron en el territorio, aunque se denunció el ingreso de éstas por parte de inmigrantes, colonos y hacendados ${ }^{28}$. Pero, al mismo tiempo, las autoridades aduaneras se esforzaban por impedir que llegaran a ser plantadas y cuando se requisaban eran quemadas junto a los embalajes ${ }^{29}$. Entre las plantas que no fueron requisadas tenemos las que importó Francisco Undurraga desde Alemania. Inició la plantación de su viña en 1891, y la variedad del Rhin la trajo en sarmientos de Bonn, Francfort, Coblenza y Colonia en tubos refrigeradores para evitar que se brotara. Una hectárea alcanzó a plantar con estas cepas auténticas del Rhin europeo, las que se fueron multiplicando y dieron origen al Rhin Undurraga ${ }^{30}$.

La contribución del Gobierno no fue sólo a través de las medidas legales sino que también con las contrataciones de profesores especialistas en el campo de la agricultura y de la vitivinicultura en particular. Entre éstos tenemos a René F. Le Feuvre que estuvo al servicio del gobierno chileno durante 30 años, desde su llegada en 1873 hasta 1903, año de su jubilación. Ocupó los cargos de director y profesor del Instituto Agrícola y de la Escuela Práctica de Agricultura de la Quinta Normal ${ }^{31}$. También estuvieron Gastón Lavergne, contratado para dirigir el Laboratorio de Patología Vegetal a partir de $1897^{32}$; Pablo Lemétayer, a cargo de la Estación Agronómica de Chile, desde 1881 hasta 1895, en que presenta su renuncia ${ }^{33}$; a inicios del siglo XX, Gastón Canu, a cargo de la Estación Enológica de Chile ${ }^{34}$. De igual forma, debemos destacar la creación de la Escuela de Viticultura y Vinificación de Cauquenes a partir de 1895 bajo la dirección de Víctor Riveros y, tras la renuncia de éste, asume el ingeniero agrónomo Manuel Rojas $\mathrm{L}^{35}$.

También el gobierno y las instituciones ligadas a la vitivinicultura se preocuparon de mejorar las condiciones para obtener éxito con la nueva actividad económica. Para ello, se formaron comisiones de estudio,

${ }^{24}$ Rojas L., Manuel 1894. "Viticultura y vinificación". Op. cit., pp. 774-805. Este autor muestra fotografias de las casas y diseños de bodegas de algunas viñas; Bengoa, José. 1990. Historia social de la agricultura chilena. Tomo II. Haciendas y campesinos. Ediciones SUR, Santiago. pp. 53-54. ${ }_{25}$ Anuario Estadístico de la República de Chile, 1861-1873; Estadística Comercial de la República de Chile, op. cit., 1844-1900.

${ }_{26}$ "Crónica agrícola: máquinas agrícolas. 1871". Boletín de la Sociedad Nacional de Agricultura. Santiago: No 7, 15 de enero. p. 109.

${ }^{27}$ Boletin de las leyes y decretos del Gobierno. Santiago: $\mathrm{N}^{\circ} 6$, junio de 1874. p. 427; $\mathrm{N}^{\circ}$ 5, mayo de 1877. pp. 304-305.

28 "Crónica agrícola: la filoxera. 1885". Boletín de la Sociedad Nacional de Agricultura. Santiago: № 18, 5 de julio. p. 380 ; Menadier, Julio. 1886. "La filoxera en el Cabo de Buena Esperanza". Boletin de la Sociedad Nacional de Agricultura. Santiago: No 16, 5 de junio. pp. 355-356.

${ }^{29}$ Memoria del Ministerio de Industria y Obras Públicas presentada al Congreso Nacional de 1888. Sociedad Nacional de Agricultura. p. XXII; Ministerio de Hacienda: Ministerio de Industrias de enero a septiembre de 1888 . Vol. 1824. Of. 3523 . Sección $1^{\circ}, \mathrm{N}^{\circ} 33$, s/p.; Ministerio de Industria y Obras Públicas: Quinta Normal de Agricultura. Vol. 1275, 1900. s/p; Diario Oficial de la República de Chile. Santiago: $N^{\circ} 6.596$, jueves 17 de mayo de 1900 . pp. 1623-1624.

${ }^{30}$ Undurraga V., Francisco R. 1943 Recuerdos de 80 años (1855-1943). Imprenta El Imparcial, Santiago. p. 105.

${ }^{31}$ Datos biográficos de los ex directores del Instituto Agrícola de Chile señores René F. Le Feuvre y Julio Besnard. Boletín de la Sociedad Agrícola del Sur. Op. cit., pp. 688-689.

${ }_{32}$ Banquete en honor de Gastón Lavergne. 1897. Boletín de la Sociedad Nacional de Viticultores. Santiago: $N^{\circ} 5$, diciembre. pp. $252-258$.

${ }^{33}$ Decreto por el que se establece una Estación Agronómica en la Quinta Normal de Agricultura. Diario Oficial de la República de Chile. Ministerio de Hacienda. Santiago: $N^{\circ} 1220$, miércoles 27 de abril de 1881. pp. 561-562; Ministerio de Hacienda, Vol. 1135, 1881. Contrato entre Alberto Blest Gana y el señor Lemétayer para hacerse cargo de la Estación Agronómica de la Quinta Normal de Agricultura; Ministerio de Industria y Obras Públicas. Escuelas de Aplicación. Consejo de Enseñanza Técnica. Quinta Normal y Sociedades de Fomento, Minería y Nacional de Agricultura. Vol. 692, 1895, tomo 2. Decreto de 27 de junio de 1895, que acepta la renuncia de Pablo Lemétayer a los cargos de director de la Estación Agronómica y de profesor de Química General del Instituto Agrícola.

${ }^{34}$ Canu, Gastón. 1909. "A los vinicultores". Boletín de la Estación Enológica de Chile. Santiago: N² 2, junio. pp. 3-4.

${ }^{35}$ Memoria que el ministro de Estado en el Departamento de Industrias y Obras Públicas presenta al Congreso Nacional en 1895. Imprenta Cervantes, Santiago, 1896. p. 18; Ministerio de Industria y Obras Públicas. Escuela de Aplicación. Consejo de Enseñanza Técnica. Quinta Normal de Agricultura y Sociedades de Fomento, Minería y Nacional de Agricultura. Vol. 692, 1895. Tomo 2, $\mathrm{N}^{\circ} 1266$, fojas 327 y No 1293 , fojas 334.

Revista de Historia, año 16, vol. 16(1), 2006, pp. 93-102 
que recorrieron la zona vitícola del país ${ }^{36}$ y otras fueron enviadas al extranjero ${ }^{37}$. Estos estudios estuvieron orientados al tratamiento de las diversas enfermedades que afectaban a las viñas chilenas, en especial se trataba de constatar la existencia de la filoxera, en qué estado se encontraban, incentivar las nuevas plantaciones con vid francesa, su cultivo, elaboración de vino y la utilización de abonos y azufre ${ }^{38}$.

El esfuerzo desplegado por los propietarios de viñas se vio recompensado a partir de la década de 1880 , pues con la incorporación de las provincias de Tarapacá y Antofagasta aumentó el mercado interno del vino chileno. A las regiones mineras se envió la mayor cantidad de vino, en especial desde el puerto de Talcahuano. Así tenemos que se enviaron desde el puerto de Valparaíso a las provincias de Tarapacá, Antofagasta, Atacama y Coquimbo, entre 1881 y 1899 , la cantidad de 5.674 .924 litros de vino blanco y 42.026 .376 litros de vino tinto y desde el puerto de Talcahuano 2.013.436 litros de vino blanco y 86.125 .315 litros de vino tinto ${ }^{39}$. Esto demuestra que aunque las viñas francesas se habían propagado en el territorio nacional no fueron suficientes como para superar la producción de la viña país. Sin embargo, al considerar el vino en botellas destinado a las provincias de Tarapacá y Antofagasta tenemos que la mayor cantidad salió desde el puerto de Valparaíso. De vino blanco fueron 947.349 litros y de vino tinto 4.355.325 litros. En cambio, desde el puerto de Talcahuano los envíos fueron menores, de vino blanco 28.044 litros y de vino tinto 457.119 litros $^{40}$. De todas formas, el mercado interno, especialmente urbano, se vio acrecentado con la incorporación de las provincias mineras del norte y la región de la Araucanía. De tal manera que se constituyó en la salvación de los viñateros en desmedro del mercado internacional, en donde los vinos chilenos tenían menor demanda e incluso era fuerte la competencia con los vinos importados, en especial, los franceses. La importación de vino blanco entre 1881-1900 fue de 6.746.377 litros y la de vino tinto de 7.038.772 litros. Mientras que la exportación de vino blanco en el mismo período fue de 496.491 litros y la de vino tinto de $6.866 .234^{41}$.

Las nuevas viñas se constituyeron en un rubro modernizador en el campo chileno, desde el punto de vista de la utilización de maquinaria y tecnología. Sin embargo, no ocurrió lo mismo con las relaciones laborales, pues se continuó utilizando a los inquilinos que vivían en las haciendas, sus mujeres e hijos y, para algunas actividades como la vendimia, también trabajaban peones forasteros ${ }^{42}$. La creación de las grandes viñas francesas se debió a la existencia de capitales provenientes de otras actividades económicas que se invirtieron en la agricultura, entre ellas, la banca, finanzas, comercio y, en especial, de la minería ${ }^{43}$.

\section{VIÑAS FRANCESAS EN CHILE CENTRAL}

La existencia de viñas francesas en Chile central no es posible conocerla debido a la falta de estadísticas que nos puedan informar al respecto en la segunda mitad del siglo XIX. Sin embargo, a través de las diversas exposiciones tanto nacionales como internacionales podemos conocer, en muchos casos, al menos los propietarios de viñas que obtuvieron reconocimientos y fueron premiados con medallas de oro, plata y bronce o con menciones honrosas. Entre ellos podemos mencionar a Silvestre Ochagavía, Magdalena Vicuña de Suberca-

\footnotetext{
${ }^{36}$ Le Feuvre, René F. 1877. "Informe sobre la enfermedad de las viñas presentado al señor Ministro de Hacienda". Boletín de la Sociedad Nacional de Agricultura. Santiago: No 11, 20 de marzo. pp. 205-213; Ibid.., "Informe sobre el estado de las viñas en San Felipe, Los Andes, Mendoza, San Juan y Buenos Aires". Boletin de la Sociedad Nacional de Agricultura. Op.cit., pp. 334-347; Idem., "Informe pasado al señor ministro de Industria y Obras Públicas sobre el estado de la agricultura y muy especialmente de la viticultura en la provincia de Maule y los medios de fomentar estas industrias". Boletín de la Sociedad Nacional de Agricultura. Santiago: N 14, 20 de julio de 1890. pp. 556-564.

${ }^{37}$ González, Eliodoro. 1889. "Informe de comisión de estudios en la República Argentina para constatar la existencia de la filoxera". Boletin del Ministerio de Industria y Obras Públicas. Santiago: No 7, julio. pp. 360-379; Le Feuvre, René F. 1889. "La misión de estudio en Europa". Boletin del Ministerio de Industria y Obras Públicas. Santiago: No 7, julio. pp. 355-360; Idem., "La misión de estudio". Boletín de la Sociedad Nacional de Agricultura. Santiago: $\mathrm{N}^{\circ}$ 4, 20 de febrero de 1890. pp. 125-130; $\mathrm{N}^{\circ} 5,5$ de marzo de 1890. pp. 164-170; №7, 5 de abril de 1890. pp. 234-238; $\mathrm{N}^{\circ} 9,5$ de marzo de 1890. pp. 316-319; No 10, 20 de mayo de 1890. pp. 354-364; No 11, 5 de junio de 1890. pp. 395-409; Rojas L., Manuel. "Informe de comisión en Europa para estudiar la viticultura, vinificación y escuelas agrícolas". Diario Oficial de la República de Chile. Santiago: $\mathrm{N}^{\circ}$ 6818,14 de febrero de 1901. pp. 475-476; №6819, 15 de febrero de 1901. pp. 491-494; $\mathrm{N}^{\circ} 6820,16$ de febrero de 1901. pp. 503-505.

${ }^{38}$ Jeria, Máximo. 1881. Informe sobre "La antracnosa en las viñas del sur de Chile". Memoria que el Ministro de Estado en el Departamento de Hacienda presenta al Congreso Nacional, 1881, pp. 45-61; La Vergne, Gastón. 1898. Informe de comisión de estudio en Talca sobre la enfermedad de las viñas. Diario Oficial de la República de Chile. Santiago: $N^{\circ} 6147$, jueves 10 de noviembre de 1898. pp. 2671-2672.

${ }^{39}$ Estadística Comercial de la República de Chile, op. cit., 1881-1899.

40 Ibid.

${ }^{41}$ Ibid.

${ }^{42}$ Salazar, Gabriel. 1989. Labradores, peones y proletarios. Ediciones SUR, Santiago, 2000. p. 164; Bengoa, José. Historia social de la agricultura chilena. Tomo II. "Haciendas y campesinos". Op. cit., pp. 52-56; Larenas Salvo, Pablo. 1910. "Monografia de la hacienda de Macul". Memoria de Titulación. Instituto Agronómico, Universidad de Chile. Santiago. pp. 93 y 95.

${ }^{43}$ Del pozo, José. Historia del vino chileno. 1998. Editorial Universitaria, Santiago. pp. 78-100.
} 
seaux, Manuel Antonio Tocornal, José Tomás Urmeneta, Miguel Collao, Juan José Arteaga, Víctor Bourgois, Juan Duprat, Guillermo Délano, Manuel Serrano, Jorge Paulsen, José Dolores Torres, Miguel Ojeda, Francisco Rojas Salamanca, Andrés Orrego, José Cabrera, L. Bunster, Roberto Lazo, Francisco Pinochet Solar, Manuel Serrano, Manuel José Benítez, Luis de la Cruz, Manuel Francisco Palacios, Servando Arteaga, Fidel S. Merino, Domingo Matte, Francisco G. Bodero, Ramón Subercaseaux, Mariano Bacarreza, Segundo Ramón González, Sres. Rogers, Serrano y Cía., Guillermo Lawrence, Alejandro Reyes, Juan Schleyer, Señores J. C. Huguet y Cía., M. Salvá de Pellé, Julio Chaigneau, Angel Gallo, Sucesión de don José Rossel, Fanny Ovalle viuda de Reyes, Carlos Ducaud, Ana Acuña viuda de Lanz, Antonio Subercaseaux, Juan Castellón, Bruno del Canto, Luis Hernández, Alfredo Masson Carrera, Guillermo Wicks, Bruno Segundo Amaral, Macario Ossa, Juan Camalez, Martín Bunster, Ricardo Solo Zaldívar, Francisco Rembadi, Olegario Alba, Maximiano Errázuriz, Luis Pereira, Rafael Mandiola, Cristián Lanz, Manuel Valenzuela, Víctor Aquiles Bianchi, Guillermo Brown, Nicolás Naranjo, Sres. Aninat e Hijos, Bernardo Dupuch, Blas Vargas, Juan Rusque, Rodulfo Montané, José Segundo del Pozo, Cámpora Hnos., J. Manuel Infante, Napoleón Meneses, Plageman Hnos., Domingo Gana, J. Ramón Gibert, Federico Varela, Margarita Marcelin viuda de Dupuch, Bruno Montel, Adolfo Eastman, Juan Despouy, Isidora Goyenechea de Cousiño, Antonio Aeta, Miguel Eyquiem, Ernesto Ducaud, Victorino Rojas Magallanes, Manuel Antonio Tocornal, Francisco Ugarte Zenteno, Guillermo Cox, Bonifacio Correa, Ricardo Waddington, José Gregorio Correa, Joaquín Díaz, Domingo Fernández Concha, Miguel Gallo Montt, Manuel Francisco Valenzuela, Federico Bodero, Baltasar Bravo, Rafael Errázuriz, Jorge Fergie, Leonidas Vial, Ismael Tocornal, M. Concha y Toro, Francisco Herboso, Luis Torres, Aracena, Navaro y Cía., Lindor Rodríguez Cañas, Fernando Chaigneau, José Tocornal, Enrique Sanfuentes, Zócimo Errázuriz, Manuel J. Díaz ${ }^{44}$.

Según los datos acerca de la existencia de viñas en el territorio nacional publicados en 1897 y elaborados por la Sociedad Nacional de Viticultores sobre la base de 161 socios propietarios, había en el país $5.031,87$ hectáreas, de las cuales $3.604,93$ correspondían a vid francesa y $1.426,94$ a la llamada país ${ }^{45}$. Comprendían desde el departamento de Freirina por el norte hasta el de Laja por el sur y claramente se observa un predominio de la variedad francesa en desmedro de la país. Destacaban entre las más grandes, considerando las que tenían una extensión superior a 40 hectáreas y, algunas menores cuyos apellidos y marcas hasta el día de hoy compiten con sus productos tanto en el mercado nacional como internacional. Por ejemplo, en el departamento de Los Andes la viña de Rafael Errázuriz U., que alcanzaba a 330 hectáreas. En el departamento de Limache, Adolfo Eastman, 54 has. En el departamento de Santiago, José Tocornal, 30 has., Luis Pereira, 45 has., Ramón Subercaseaux, 80 has., Nicolás Valdivieso, 31 has., Silvestre Ochagavía, 40 has., Isidora Goyenechea de Cousiño, 71,87 has. En el departamento de Victoria, Francisco Undurraga, 70 has. (10 has. correspondían a cepa país), Ismael Tocornal, 30 has. En el departamento de Maipo, Daniel Concha ("Concha y Toro"), 60 has. En el departamento de Lontué, Bonifacio Correa, 70 has $^{46}$ (para otras viñas ver Cuadro I).

\footnotetext{
${ }^{44}$ Briones Quiroz, Félix. 1995. "Vitivinicultura chilena: 1850-1900". Tesis de Magíster en Historia. Universidad de Santiago de Chile. pp. 184-197. Apéndice II. Exposiciones nacionales e internacionales y premios obtenidos por los vinos chilenos. Las exposiciones nacionales corresponden a las realizadas en los años $1863,1869,1872,1881,1882,1884,1888$ y, las internacionales, a los años $1875,1876,1882,1886,1888,1889,1897$.

45 Memoria del Ministerio de Industria y Obras Públicas presentada al Congreso Nacional en 1897. Imprenta de La Patria, Valparaiso, 1897. Segunda memoria de la Sociedad Nacional de Viticultores. pp. 155-163. Entre las variedades país estaban: uva de gallo, italia negra y blanca, San Francisco, negra común; variedades francesas de uva blanca: pinot blanco, gamet blanco, blanquette, chasselas de Fontainebleau, sauvignon, semillón, y de uva negra: cabernet sauvignon, gamet negro, malbeck, cot-rouge, merlot, meunier, verdot. Ver: Séve, Eduardo. "La patria chilena". Boletín de la Sociedad Nacional de Agricultura. Op.cit., pp. 546-547; Rojas L., Manuel. 1950. Viticultura y vinificación. Nascimento, Santiago. pp. 56-95.

${ }^{46}$ Memoria del Ministerio de Industria y Obras Públicas presentada al Congreso Nacional en 1897. Op. cit., pp. 156-161.
} 
Cuadro I: viñas superiores a 40 has. en 1897.

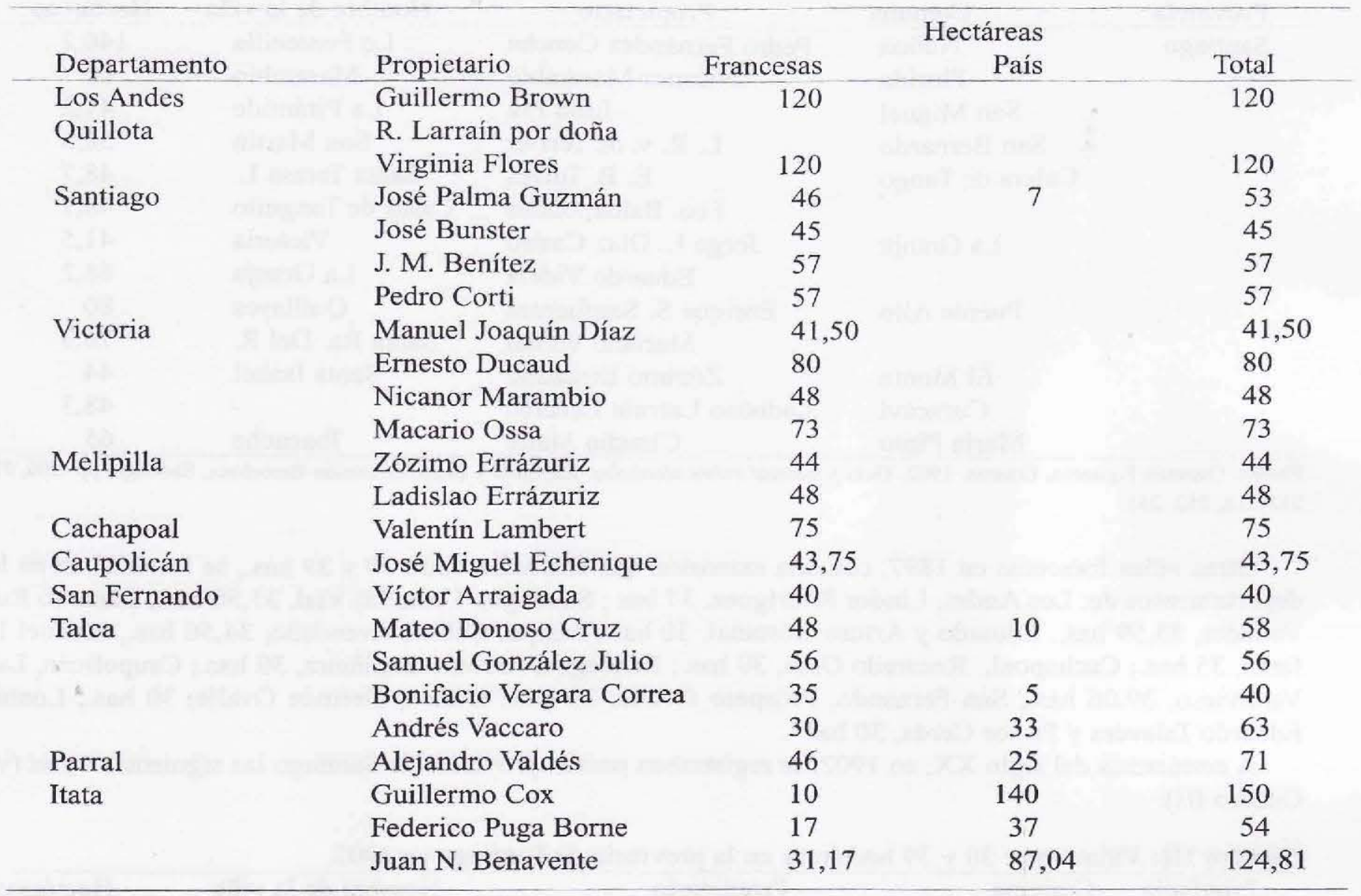

Fuente: Memoria del Ministerio de Industria y Obras Públicas presentada al Congreso Nacional en 1897. Imprenta de La Patria, Valparaíso, 1897. Segunda memoria de la Sociedad Nacional de Viticultores. pp. 156-161.

Hacia 1902, en otra estadística vitivinícola nacional, además del propietario, extensión y departamento, se mencionaban la provincia, comuna y el nombre de la viña, pero sin diferenciar si eran francesas o país. Por ejemplo, entre las superiores a 40 hectáreas existían: en la provincia de Valparaíso, departamento de San Felipe, comuna Panquehue, Rafael Errázuriz U., "Panquehue", 460 has. En el departamento de Limache, comuna Limache Alto, Adolfo Eastman, "Urmeneta", 50 has. En la provincia de Santiago, departamento de Santiago, comuna de San Miguel, Silvestre Ochagavía, "Ochagavía", 50 has., Ramón Subercaseaux, "Subercaseaux", 80 has., M. Alberto Valdivieso, "Santa Elena", 60 has., Luis Pereira, "Santa Carolina", 50,3 has. En el departamento de Victoria, comuna de San Bernardo, Joaquín Tocornal, "San Joaquín", 40,7 has.; comuna de Talagante, Francisco Undurraga, 45 has. (Cuadro II). En la provincia de Talca, departamento de Lontué, comuna Molina, Francisco Javier Correa, "Lontué", 75 has. y Dussaillant Hnos, "Casa Blanca", 43 has ${ }^{47}$.

\footnotetext{
${ }^{47}$ Guzmán Figueroa, Erasmo. 1902. Guia y manual sobre alcoholes. Imprenta y Encuadernación Barcelona, Santiago. pp. 206, 210, 212-218, 252, 261. De acuerdo a esta fuente desde la provincia de Atacama por el norte a la de Cautín por el sur, existían alrededor de 4.200 propietarios de viñas. Sin embargo, el editor señala que "la Guía de los viñedos es incompleta por culpa de los mismos propietarios, que no han prestado la debida atención a nuestras circulares".
} 
Cuadro II: Viñas superiores a 40 has. en la provincia de Santiago en 1902.

\begin{tabular}{rrrrr} 
Provincia & Comuna & Propietario & Nombre de la viña & Hectáreas \\
\cline { 3 - 5 } Santiago & Nuñoa & Pedro Fernández Concha & Lo Fontecilla & 146,2 \\
Florida & Nicanor Marambio & Marambio & \multicolumn{1}{c}{60} \\
San Miguel & Julio Prá & La Pirámide & 41,2 \\
San Bernardo & L. R. v. de Terrier & San Martín & 58,5 \\
Calera de Tango & E. B. Torres & Santa Teresa L. & 48,7 \\
& Fco. Bahamondes & Casas de Tanguito & 48,7 \\
La Granja & Jorge L. Díaz Castro & Victoria & 41,5 \\
& Eduardo Videla & La Granja & 68,2 \\
Puente Alto & Enrique S. Sanfuentes & Quillayes & 80 \\
& Mariano Vernal & Santa Ra. Del R. & 76,3 \\
El Monte & Zózimo Errázuriz & Santa Isabel & 44 \\
Curacaví & Ladislao Larraín Lecaros & - & 48,3 \\
María Pinto & Claudio Matte & Ibacache & 65
\end{tabular}

Fuente: Guzmán Figueroa, Erasmo. 1902. Guia y manual sobre alcoholes. Imprenta y Encuadernación Barcelona, Santiago. pp. 206, 210, $212-218,252,261$.

Otras viñas francesas en 1897 , con una extensión que fluctuaba entre 30 y 39 has., sc localizaban en los departamentos de: Los Andes, Lindor Rodríguez, 37 has.; Santiago, Leonidas Vial, 31,50 has., Eduardo Ruiz Valledor, 33,59 has., Eduardo y Arturo Costabal, 30 has.; Maipo, Calixto Avendaño, 34,50 has., Manuel Infante, 35 has.; Cachapoal, Recaredo Ossa, 30 has.; Rancagua, Antonio Espiñeira, 30 has.; Caupolicán, Luis Valdivieso, 39.06 has.; San Fernando, Próspero Ovalle, 30 has.; Curicó, Germán Ovalle, 30 has.; Lontué, Eduardo Talavera y Pastor Cerda, 30 has $^{48}$.

A comienzos del siglo XX, en 1902, se registraban para la provincia de Santiago las siguientes viñas (ver Cuadro III):

Cuadro III: Viñas entre 30 y 39 hectáreas en la provincia de Santiago en 1902.

\begin{tabular}{lllll}
\hline Provincia & Comuna & \multicolumn{1}{c}{ Propietario } & Nombre de la viña & Hectáreas \\
\hline Santiago & Recoleta & E. Aguirre Luco & Conchalí & 30 \\
& & J. Gabriel Palma & - & 38 \\
& Nuñoa & Prudencio Armijo & Los Guindos & 36,2 \\
& & Guillermo Aguirre & Conchalí & 39 \\
& & Recoleta Dominica & Apoquindo & 31,5 \\
& & Eugenio Guzmán Montt & S. R. de Apo. & 32,9 \\
& Quilicura & Pedro Briceño & Campinito & 30,8 \\
Maipú & Roberto Brieba & San Andrés & 30 \\
San Bernardo & Luis Corti & Santa Teresa & 36 \\
& & - & 32,5 \\
& Pedro Donoso Vergara & - & 39 \\
& Renca & Ismael Tocornal & El Mirador & 31,2 \\
Talagante & V. M. Moreno & S. Vte. de M. & 32,5 \\
& Ramón Undurraga & San Vicente & 37,7 \\
Las Condes & L. W. Urtisbondo & Lo Matta & 31,6 \\
Las Lomas & Enrique Varas Palacios & Ricardo Vial & Lo Prado & 30 \\
Puente Alto & José Luis Coo & San Carlos & 30 \\
& Eduardo Charme & Miraflores & 37 \\
Colina & José Tocornal & El Algarrobal & 31,2 \\
La Florida & Manuel Zavala & Tarapacá & 30 \\
La Granja & Víctor Faure & Toldería & 30 \\
San Miguel & Nicanor Opazo Silva & Chicureo & 34,5 \\
Calera de Tango & Antonio Celsi & - & 30,9
\end{tabular}

Fuente: Guzmán Figueroa, Erasmo. 1902. Guía y manual sobre alcoholes. Imprenta y Encuadernación Barcelona, Santiago. pp. $212-217$.

48 Memoria del Ministerio de Industria y Obras Públicas presentada al Congreso Nacional en 1897. Op. cit., pp. 155-159.

Revista de Historia, año 16, vol. 16(1), 2006, pp. 93-102 
La existencia de variedades francesas podemos apreciarla en algunas viñas, por ejemplo, en el departamento de la Victoria, la viña de E. Cotidio Fernández tenía una extensión de 14 cuadras, de las cuales 8 eran de Cabernet, Cot, Semillón, Pinot, Torrontes y de la variedad país había 6 cuadras; Francisco Undurraga era propietario de una viña de 70 has, correspondiendo 60 a vid francesa, distribuidas en 20 de Cabernet, 20 de Pinot, 20 de Semillón blanco; y 10 a la viña país; Enrique Morandé, poseía 28 cuadras, 14 eran de Cot, Semillón, Merlot, Cabernet y las otras 14 eran de viña país; Victorino Rojas Magallanes, con 14 cuadras de Cabernet, Merlot, Cot, Semillón, Verdot. En el departamento de San Fernando, Víctor Arriagada tenía 40 cuadras de variedades francesas: Cabernet, Cot, Pinot, Romana, Semillón, Saint Emilion, Loca Blanca, Merlot, Chasselas. En el departamento de Lontué, Bonifacio Correa era propietario de 70 has., siendo dominante la variedad Cabernet con 59,5 has. y el resto eran de Romana, Cot-Rouge, Pinot blanco, Moscatel, Semillón blanco; Eduardo Talavera tenía 30 has, repartidas en 12 de Cabernet, 6 Semillón blanco, 4 Cot-Rouge, 4 Pinot negro, 4 Merlot $^{49}$. También podemos mencionar la viña "Urmeneta", en Limache, con sus variedades Cabernet, Pinot, Romana, Tressot, Cot-rouge, Malbeck, Morillon blanco, Petit Verdot; la viña "San Andrés" de Juan Despouy, en Santiago, tenía Pinot, Cabernet, Cot, Semillón blanco y Tintorero; la viña "Santa Carolina", de Luis Pereira, en Santiago, con 45.000 plantas de Cabernet, 25.000 de Cote-rouge, 5.000 de Semillón, 30.000 de Pinot negro, 70.000 de Pinot y Gamet, 25.000 de Romana; la viña "Arnut", de Ernesto Ducaud, en San Bernardo, con sus plantaciones de Semillón blanco, Loca blanca, Cabernet, Merlot, Verdot, Cote-rouge, Pinot blanco ${ }^{50}$.

\section{CONCLUSION}

La creación de las viñas francesas en Chile central contribuyó a la diversificación de la actividad económica en el campo chileno en la segunda mitad del siglo XIX, período en el cual se sentaron las bases de la nueva vitivinicultura chilena.

Contribuyeron a la consolidación de esta nueva actividad los esfuerzos desplegados por los particulares que realizaron inversiones en este rubro, las medidas legales que prohibieron la entrada de vid extranjera al país y el aporte de los franceses que en la práctica hicieron posible la idea de convertir a Chile en la Francia de América del Sur.

A pesar de que en Chile se producían vinos de calidad, siguiendo las reglas de producción aplicadas en Francia, el mercado externo fue de escasa importancia y no se cumplieron las expectativas comerciales a que aspiraban los productores nacionales. Aunque los especialista franceses reconocían que desde Chile se exportaba buen vino, al mismo tiempo manifestaban que no poseía la calidad suficiente para competir con los vinos elaborados en Francia.

Esta nueva actividad en el campo de Chile central requirió de grandes capitales que fueron aportados por otros rubros de la actividad económica nacional, en especial la minería. Pues, la contratación de un diseñador para las casas, parques y bodegas subterráneas, y la construcción de los mismos requería de fuertes sumas de dinero. Además, para los trabajos de plantación, cultivo de las plantas de vid y elaboración de vino se requería de un especialista francés que era contratado para tal efecto. De igual forma, debía solventarse la adquisición de maquinaria, herramientas y utensilios que hacían posible la realización de los diversos trabajos en las nuevas viñas.

Cabe destacar que la vitivinicultura nacional fue una de las industrias más prósperas del país, debido a los capitales invertidos y al número de trabajadores ocupados, estimados en $40.000^{51}$.

Si bien es cierto que la vitivinicultura experimentó una "verdadera revolución" en la región central de Chile a partir de mediados del siglo XIX, no sólo se debió a los grandes propietarios de viñas francesas de los cuales aún se conservan los apellidos que identifican a los viñedos. Al mismo tiempo, debemos destacar a los pequeños propietarios que también plantaron estas variedades, pero que no perduraron en el tiempo, tanto por la competencia de los grandes propietarios de las nuevas viñas como la importación de vino, especialmente de Francia, que eran comercializados en el mercado interno.

\footnotetext{
49 "Actas de la Sociedad Nacional de Viticultores". Boletin de la Sociedad Nacional de Viticultores. Santiago: N 1, 5 de julio de 1897. pp. 27-28.

${ }^{50}$ Rojas L., Manuel. Tratado de viticultura y vinificación. Op. cit., pp. 41-42

${ }^{51}$ Memoria del Ministerio de Industria y Obras Públicas, 1896. p. 119.
} 


\section{FUENTES Y BIBLIOGRAFIA}

\section{FUENTES}

Anuario Estadístico de la República de Chile, 1861-1873.

Boletín del Ministerio de Industria y Obras Públicas, 1889.

Boletín de la Estación Enológica de Chile, 1909.

Boletin de la Sociedad Agricola del Sur, 1904.

Boletín de la Sociedad de Fomento Fabril, 1884

Boletín de la Sociedad Nacional de Agricultura, 1870-1894

Boletín de la Sociedad Nacional de Viticultores, 1897

Boletín de las leyes y decretos del Gobierno, 1874, 1877.

Diario Oficial de la República de Chile, 1881, 1898, 1901

Estadistica Comercial de la República de Chile, 1844-1900.

Memoria del Ministerio de Hacienda, 1881.

Memoria del Ministerio de Industria y Obras Públicas, 1888, 1895, 1896, 1897.

Memoria del Ministerio de Relaciones Exteriores y Colonización, 1874.

Ministerio de Hacienda: Ministerio de Industrias de enero a septiembre de 1888, 1888.

Ministerio de Hacienda: Sociedad Nacional de Agricultura, 1881.

Ministerio de Industria y Obras Públicas: Escuelas de Aplicación. Consejo de Enseñanza Técnica. Quinta Normal y Sociedades de Fomento, Minería y Nacional de Agricultura, 1895.

Ministerio de Industria y Obras Públicas: Quinta Normal de Agricultura, 1900.

\section{BIBLIOGRAFIA}

BENGOA, José. 1990. Historia social de la agricultura chilena. Tomo II. Haciendas y campesinos. Ediciones SUR, Santiago.

BRIONES QUIROZ, Félix. 1995. "Vitivinicultura chilena: 1850-1900”. Tesis de Magíster en Historia. Universidad de Santiago de Chile

DEL POZO, José. 1998. Historia del vino chileno. Editorial Universitaria, Santiago.

GUZMAN FIGUEROA, Erasmo. 1902. Guia y manual sobre alcoholes. Imprenta y Encuadernación Barcelona, Santiago.

ROJAS L., Manuel. 1891. Tratado de viticultura y vinificación. Imprenta de "La Libertad", Talca.

ROJAS L., Manuel. 1897. Tratado de viticultura y vinificación. Imprenta y Librería Ercilla, Santiago.

ROJAS L., Manuel. 1950. Tratado de viticultura y vinificación. Nascimento, Santiago.

SALAZAR, Gabriel. Labradores, peones y proletarios. 2000. LOM Ediciones, Santiago.

TORNERO, Recaredo S. Chile Ilustrado. 1872. Guia descriptivo del territorio de Chile, de las capitales de provincia y de los puertos principales. Librería y Agencias del Mercurio, Valparaíso.

UNDURRAGA V., Francisco R. Recuerdos de 80 años (1855-1943). 1943. Imprenta El Imparcial, Santiago. 